Weiterbildung

\section{Palliative Care in der Kinderkrankenpflege}

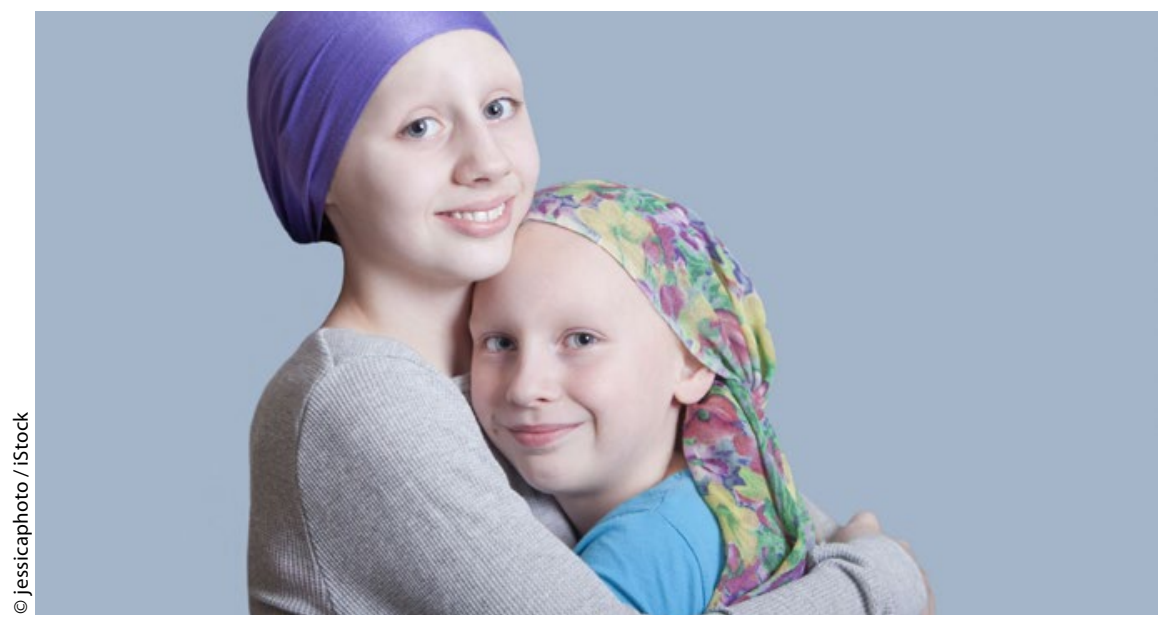

Bereits jetzt ist die Anmeldung zur Weiterqualifizierung zur Pflegekraft für Palliative Care in der Gesundheits- und Kinderkrankenpflege für das kommende Jahr möglich. In diesem Basiskurs Palliativversorgung in der Kinderkrankenpflege sollen die Grundlagen von Palliative Care praxisbezogen so vermittelt werden, dass eine Umsetzung in den beruflichen Alltag gut möglich ist. Die Weiterbildung dauert eine Woche (40 Stunden auf fünf Arbeitstage verteilt) und findet vom 26. bis 30. September 2016 in der Diakoniestation Frankfurt a.M. statt. Die Kursge- bühren betragen $895 €$ pro Teilnehmer. Der Lehrgang richtet sich an Kindergesundheitsund Kinderkrankenpflegerinnen/pfleger oder sonstige Interessierte nach Prüfung der Voraussetzungen.

Notwendig ist ein Nachweis über mindestens ein Jahr Berufspraxis. Nach erfolgreichem Abschluss erhalten die Teilnehmer ein Zertifikat des B.F.G. und der DGP e.V. Veranstalter ist das Bildungsinstitut Fachbereiche Gesundheitswesen B.F.G.

www.bfg-kray.de
Hausengel-Akademie

Schulungsangebot für Betreuungskräfte

\begin{abstract}
— Pflegebedürftige mit demenziellen Veränderungen, psychischen Erkrankungen oder geistigen Behinderungen haben in der Regel hohen Betreuungsbedarf. Um diesen Anforderungen gerecht zu werden, bietet die Hausengel Betreuungsdienstleistungen $\mathrm{GmbH}$ ihren Franchisenehmern seit 2011 Aus- und Weiterbildungen an ihrem hauseigenen, staatlich anerkannten Weiterbildungsträger an. Die im letzten Jahr eingeführte Ausbildung zum Betreuungsassistenten nach §87b SGB XI wird nun auch externen Interessierten angeboten. Das Angebot richtet sich insbesondere an osteuropäische Betreuungskräfte, die selbstständig in diesem Bereich tätig sein möchten. Sie umfasst drei Module mit insgesamt 285 Stunden. „Betreuungskräfte müssen sich mit Krankheiten wie Demenz oder Parkinson auskennen, sie müssen wissen, wie sie sich Erkrankten gegenüber verhalten sollten und in Notsituationen eigenverantwortlich und richtig reagieren", macht Simon Wenz, Geschäftsführer und Gründer der Hausengel Akademie seine Bemühungen deutlich.
\end{abstract}

www.hausengel.de/hausengel-akademie

\title{
Pflegemanagement-Award 2016 für Nachwuchsführungskräfte
}

— Ab sofort können Nachwuchsführungskräfte in der Pflege für den Pflegemanagement-Award 2016 nominiert werden. Die Preisverleihung findet im Rahmen des von Springer Medizin veranstalteten Kongresses "Pflege 2016" in Berlin statt. Der Pflegemanagement-Award feiert 2016 ein kleines Jubiläum: Zum fünften Mal in Folge wird eine Nachwuchsführungskraft für ihre Leistungen mit dem Pflegemanagement-Award ausgezeichnet werden. Noch bis zum 19. Oktober 2015 können Führungskräfte in der Pflege ihren Nachwuchs für den Preis vorschlagen.
Initiator des Preises ist der Bundesverband Pflegemanagement. Hauptsponsor ist auch dieses Jahr ZeQ AG, eine der führenden Unternehmensberatungen für Krankenhäuser, Rehakliniken und Psychiatrien. „Die überaus positive Resonanz und die vielen herausragenden Nominierungen zeigen, dass wir mit dem Pflegemanagement-Award die richtige Form der Anerkennung für die tolle Leistung des Nachwuchses in der Öffentlichkeit gefunden haben", so Peter Bechtel, Vorstandsvorsitzender des Bundesverbands Pflegemanagement. Gesucht werden

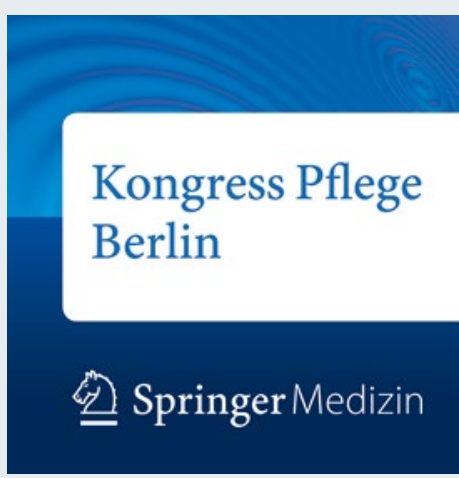

Nachwuchsführungskräfte, die sich mit Engagement und Kompetenz für die Stärkung der Position ihrer Profession einsetzen.

www.bv-pflegemanagement.de 